\title{
Batten Disease
}

National Institute of Neurological Disorders and Stroke (NINDS)

\section{Source}

National Institute of Neurological Disorders and Stroke (NINDS). Batten Disease

Information Page.

Batten disease is the name for a group of inherited nervous system disorders that most often begin in childhood and interfere with a cell's ability to recycle a cellular residue called lipofuscin. Batten is commonly being used to describe the many forms of the disease, called neuronal ceroid lipofuscinosis. The many forms of the disease are classified by the gene that causes the disorder, with each gene being called CLN (ceroid lipofucinosis, neuronal) and given a different number as its subtype. Because of the different gene mutations, signs and symptoms range in severity and progress at different rates.

Symptoms generally include:

- progressive vision loss leading to blindness,

- seizures,

- movement disorder, and

- dementia.

Developmental skills such as standing, walking, and talking may not be achieved or are gradually lost. Other symptoms that continue to worsen over time include learning difficulties, poor concentration, and progressive loss of language skills and speech. Most children become bedridden and unable to communicate. Some children develop problems sleeping. Currently, most diagnoses of Batten disease are made by genetic testing. 